\title{
A CASE CONTROL STUDY TO EVALUATE THYROID DYSFUNCTION AS A RISK FACTOR FOR DIABETIC RETINOPATHY IN TYPE 2 DIABETES MELLITUS
}

\author{
${ }_{1}^{1}$ Associate Professor, Department of Ophthalmology, Madurai Medical College. \\ 2Professor, Department of Ophthalmology, Madurai Medical College. \\ ${ }^{3}$ Senior Resident, Department of Ophthalmology, Madurai Medical College. \\ ${ }^{4}$ Senior Resident, Department of Ophthalmology, Madurai Medical College. \\ 5Junior Resident, Department of Ophthalmology, Madurai Medical College.
}

Subbaiah Vasan Chandrakumar1, Ponnusamy Thiyagarajan², Amit K. Jain³, Thangaraj Murugalakshmi4, Srinivasan Muralikrishnan ${ }^{5}$

\begin{abstract}
This is a Case Control Study that was conducted among 100 Type 2 DM (Diabetes Mellitus) patients who attended the OP and the wards of Ophthalmology Department at Government Rajaji Hospital, Madurai, to assess the prevalence of thyroid dysfunction among Type 2 DM patients and whether thyroid dysfunction is associated with increased risk of retinopathy in Type 2 diabetes patients. Patients diagnosed with Type 2 DM on treatment for more than 5 years of diabetes mellitus and over 12 years of age were taken up for this study. Patients with known history of thyroid disorder, patients on drugs known to affect thyroid function like lithium, amiodarone, oral contraceptive pills, etc., pregnant mothers, systolic blood pressure more than $150 \mathrm{~mm}$ of $\mathrm{Hg}$ and diastolic more than $90 \mathrm{~mm}$ of $\mathrm{Hg}$, with nephropathy and those patients for whom fundus examination was not possible, patients not consenting for this study were excluded from this study. Data and blood samples will be collected from subjects and serum shall be extracted for thyroid profile. All the study patients will undergo a detailed ocular examination for grading of Diabetic Retinopathy (DR) as per the Early Treatment Diabetic Retinopathy Study (ETDRS) guidelines.
\end{abstract}

\section{RESULTS}

Odds Ratio (OR) for determining association between risk factor and disease was 5.06, with an upper 95\% Confidence Limit of 15.051 and a lower confidence limit of 1.703, which means the odds of a diabetic patient with thyroid dysfunction (subclinical and overt hypothyroidism) developing retinopathy is 5.06 times that of a diabetic patient without thyroid dysfunction. According to this study the prevalence of thyroid dysfunction among the 100 Type 2 Diabetes Mellitus patients studied was $23 \%$ with the most common problem being Subclinical Hypothyroidism (SCH) at $21 \%$ among the study population followed by overt hypothyroidism at $2 \%$. As expected the prevalence of thyroid dysfunction ${ }^{3}$ among females (32\%) was much more than among males (14\%), the odds ratio being 5.06. Chi-Square statistic applied to our data revealed P-value of $<0.005$.

\section{CONCLUSION}

Our study showed a significant association between subclinical and overt hypothyroidism with development of diabetic retinopathy in this study population.

\section{KEYWORDS}

Hypothyroidism, Diabetic Retinopathy, Type 2 Diabetes Mellitus.

HOW TO CITE THIS ARTICLE: Chandrakumar SV, Thiyagarajan P, Jain AK, et al. A case control study to evaluate thyroid dysfunction as a risk factor for diabetic retinopathy in type 2 diabetes mellitus. J. Evolution Med. Dent. Sci. 2016;5(72):5253-5256, DOI: $10.14260 /$ jemds/2016/1190

\section{INTRODUCTION}

Diabetes Mellitus is the most common endocrine disorder world over and India is considered the world capital of this disease. Type 2 DM being the most common type by 2030 , diabetes would be the $7^{\text {th }}$ leading cause of death out of which $80 \%$ of these deaths would occur in developing and under developed countries. Type 2 DM affects nearly every physiological process in the body, hence it has wide ranging systemic effects.

Diabetic Retinopathy is an important and most common cause of blindness in the developed world. The disease is characterised by progressive changes in retinal

Financial or Other, Competing Interest: None.

Submission 01-08-2016, Peer Review 25-08-2016,

Acceptance 31-08-2016, Published 07-09-2016.

Corresponding Author:

Dr. Subbaiah Vasan Chandrakumar,

Eye Department Op. No. 50,

Govt. Rajaji Hospital, No. 1, Panagal Road,

Madurai-625020, Tamilnadu.

E-mail: eyedrck@gmail.com

DOI: $10.14260 /$ jemds $/ 2016 / 1190$ microvasculature leading to retinal hypoperfusion, increased vascular permeability and neovascularisation along with Clinically Significant Macular Oedema (CSME) causing severe and permanent blindness.

Thyroid disorder is the common endocrine problem second only to diabetes.

Most of the cells in our body need the thyroid hormone for functioning properly as it has effects on a significant number of physiological processes. Moreover, it is not unusual for a patient to be affected by both Type 2 DM and thyroid disorders.

There are varying reports regarding the prevalence of thyroid dysfunction among diabetics, most common thyroid abnormality being Subclinical Hypothyroidism (SCH). Diabetic patients have an increased frequency of retinopathy and nephropathy when associated with subclinical hypothyroidism. At the same time, the severity of retinopathy in these patients is said to be more. Even subclinical hypothyroidism has a negative impact on lipid metabolism and it is an independent risk factor for myocardial infarction and retinopathy. 
The two conditions have strong association ${ }^{1}$ and have important clinical implications for treatment and insulin sensitivity. Impaired renal clearance is said to be an important cause of the reduction in insulin requirements in hypothyroidism. Decreased gluconeogenesis makes these patients highly prone for hypoglycaemia.

Another possible mechanism linking subclinical hypothyroidism ${ }^{2}$ to vascular diseases is the finding that thyroid hormones inhibit collagen-induced platelet aggregation and directly relax smooth muscles. Further hypothyroidism is accompanied by a hypercoagulable state and increased blood viscosity. Hyperthyroidism is seen less commonly in Diabetes, but is associated with higher risk of development of potentially life-threatening ketoacidosis.

Therefore, early detection and correction of thyroid abnormalities is essential for proper diabetic control as well as for prevention of diabetic complications as it may greatly increase the quality of life in these patients. At the molecular level, thyroid hormone has cell surface receptors for integrin $\alpha v \beta 3$ that mediates the angiogenic activity of thyroid hormones and integrin $\alpha \nu \beta 3$ has proangiogenic effects on growth factors implicated in the pathogenesis of retinal new vessels, 3 including erythropoietin, growth hormone, Basic Fibroblast Growth Factor (bFGF), insulin-like growth factor-1 (IGF-1) and Vascular Endothelial Growth Factor (VEGF).

Thyroid hormone has well-documented effects on new vessel formation. These effects are mediated via integrin receptor for the thyroid hormones on the vascular smooth muscle cells and endothelial cells reflecting the relation between genomic and non-genomic mechanisms invoked by the thyroid hormones.

\section{METHODS}

A case control study was conducted among 100 Type 2 DM patients attending the OP and wards at Government Rajaji Hospital, Madurai. Subjects were evaluated for entry into the study if they are 12 years of age or older. Subjects believed to fulfil all eligibility criteria and none of the exclusion criteria were invited to participate in the study.

\section{Inclusion Criteria}

1. Patients diagnosed with Type 2 Diabetes on treatment.

2. Duration $>5$ years of diabetes mellitus.

3. Age $>12$ yrs.

\section{Exclusion Criteria}

1. Patients with known history of thyroid disorder prior to Type 2 DM.

2. Patients on drugs known to affect thyroid function like lithium, amiodarone, oral contraceptive pills, etc.

3. Pregnant mothers.

4. Patients in whom fundus cannot be examined.

5. Patients not consenting for the study.

6. Systolic BP $>140 \mathrm{~mm}$ of $\mathrm{Hg}$ and Diastolic $\mathrm{BP}>90 \mathrm{~mm}$ of $\mathrm{Hg}$.

7. Glaucoma patients.

8. Nephropathy patients.

\section{Ethical Committee Clearance}

Ethical committee clearance was obtained from Institutional Review Board/Independent Ethics Committee of Government Rajaji Hospital.

\section{Assessment of Thyroid Profile}

Data and blood samples collected from established cases of Type 2 DM patients on treatment from which serum was extracted for Thyroid Profile - T3, T4 and TSH levels was estimated from the serum samples of the selected patients by ELISA technique.

\section{The following Guidelines for Detection of Thyroid} Dysfunction were considered

- Normal-when T3, T4 and TSH were within the normal.

- Primary hypothyroidism-when TSH is super normal and T3, T4 are subnormal.

- Primary hyperthyroidism-when TSH is subnormal and T3, T4 are super normal.

- Subclinical hypothyroidism-when TSH is super normal and T3, T4 are normal.

- Subclinical hyperthyroidism-when TSH is subnormal and T3, T4 are normal.

\begin{tabular}{|c|c|c|}
\hline Test & Abbreviation & Typical Ranges \\
\hline Serum Thyroxin & $\mathrm{T} 3$ & $4.6-12 \mathrm{ug} / \mathrm{dL}$ \\
\hline $\begin{array}{c}\text { Serum } \\
\text { Triiodothyronine }\end{array}$ & $\mathrm{T} 4$ & $80-180 \mathrm{ng} / \mathrm{dL}$ \\
\hline $\begin{array}{c}\text { Serum Thyroid- } \\
\text { Stimulating } \\
\text { Hormone }\end{array}$ & $\mathrm{TSH}$ & $0.5-6 \mathrm{IU} / \mathrm{mL}$ \\
\hline
\end{tabular}

\section{Assessment of Diabetic Retinopathy}

A detailed ocular examination was performed in all the study patients for detection and grading of DR by an ophthalmologist. The prevalence of hypothyroidism in patients with and without diabetic retinopathy was analysed.

ETDRS has classified Non-Proliferative Diabetic Retinopathy (NPDR) into mild, moderate, severe and very severe and Proliferative Diabetic Retinopathy (PDR) into early PDR and high-risk PDR. This is as follows:

A. Mild NPDR: Presence of at least one microaneurysm, definition not met for B, C, D, E or F.

B. Moderate NPDR: Haemorrhages and/or microaneurysm, presence of soft exudates, venous beading, Intraretinal Microvascular Abnormality (IrMA) definitely present, definition not met for C, D, E or F.

C. Severe NPDR: Haemorrhages and 1 or more microaneurysm in all four quadrants or venous beading in two or more quadrants or IrMA >standard photo 8A in at least one quadrant, definition not met for $\mathrm{D}, \mathrm{E}$ or $\mathrm{F}$.

D. Very severe NPDR: Any two or more of the changes seen in severe NPDR, definition not met for $\mathrm{E}$ or F.

E. Early PDR: Presence of new vessels, definition not met for F.

F. High-risk PDR: Includes any of the following characteristics-Neovascularisation of the Disc (NVD) $>1 / 3$ rd to $1 / 4$ th disc diameter, NVD $<1 / 3$ rd to $1 / 4$ th disc diameter with vitreous/preretinal haemorrhage, Neovascularisation Elsewhere (NVE) with vitreous/preretinal haemorrhage, High-Risk Characteristics (HRC) were defined by Diabetic Retinopathy Study (DRS), as the patient if not treated urgently is at a high risk of severe visual loss.

G. Diabetic maculopathy: 3 types 
- Focal Maculopathy- When microaneurysm, haemorrhage, macular oedema, hard exudates arranged in a circinate pattern is present.

- Diffuse Maculopathy-Presence of diffuse retinal oedema and thickening.

- Ischaemic Maculopathy-Marked visual loss with microaneurysms, haemorrhage, mild or no macular oedema.

H. Advanced eye disease: Persistent or recurrent vitreous haemorrhage, Traction retinal detachment, and Neovascular glaucoma.

\section{Observation and Analysis}

The age of the patients studied varied from 37 years to 78 years. Among the 100 patients, 50 were males and 50 were females. The duration of diabetes in our study patients was between 5 years and 20 years ( 72 were between 5 to 10 yrs., 19 were between 10 to 15 yrs. and 9 were between $15-20$ yrs.)

Thyroid dysfunction was seen in 23 patients $^{4}$ (7 were males and 16 were females). Among the 50 patients with retinopathy, 18 had some form of thyroid dysfunction while among the remaining 50 patients without retinopathy, thyroid dysfunction was seen in 5 . The most common form being Subclinical Hypothyroidism (SCH), which was seen in 21 patients. 2 patients had frank hypothyroidism. Diabetic retinopathy was seen in 50 patients, 46 had NPDR while 4 had PDR. Among them, 28 were males and 22 were females.

According to this study, the prevalence of thyroid dysfunction ${ }^{5}$ among the 100 Type 2 Diabetes Mellitus patients studied was $23 \%$ with the most common problem being Subclinical Hypothyroidism ( $\mathrm{SCH}$ ) at $21 \%$ among the study population followed by overt hypothyroidism at $2 \%$. As expected, the prevalence of thyroid dysfunction among females (32\%) was much more than among males (14\%).

Odds Ratio (OR) for determining association between risk factor and disease was 5.06 with an upper 95\% Confidence Limit of 15.051 and a lower confidence limit of 1.703, which means the odds of a diabetic patient with thyroid dysfunction (subclinical and overt hypothyroidism) developing retinopathy is 5.06 times that of a diabetic patient without thyroid dysfunction developing the same.

Chi-square statistic applied to our data revealed P-value of $<0.005$. Our study showed a significant association between subclinical and overt hypothyroidism and diabetic retinopathy in the study population.

In our study, the number of patients with PDR was only 4 and this number was too less to study an association between thyroid dysfunction and severity of retinopathy.

\section{DISCUSSION}

The different mechanisms by which thyroid dysfunction affect the Diabetes status are

1. Thyroid dysfunction, both hypothyroidism and hyperthyroidism are associated with increased insulin resistance.

2. In hypothyroidism, the renal clearance of insulin is decreased resulting in reduced insulin requirements.

3. The loss of appetite seen in hypothyroidism contributes to decreased insulin requirements.

4. Type 1 Diabetic patients who have Hypothyroidism have reduced rate of gluconeogenesis with an increased incidence of hypoglycaemia.
5. Even subclinical hypothyroidism has a negative impact on lipid metabolism and it is an independent risk factor for myocardial infarction and retinopathy.

6. Another possible mechanism linking $\mathrm{SCH}$ to vascular diseases is the finding that thyroid hormones inhibit collagen-induced platelet aggregation 6 and directly relax smooth muscles.

7. Further hypothyroidism is accompanied by a hypercoagulable state and increased blood viscosity.

Thus, presence of associated thyroid dysfunction has significant implications when it comes to glycaemic control, insulin resistance, glycaemic control and complications of diabetes. Patients with associated hypothyroidism have been noted to have a higher incidence of microvascular complications especially nephropathy and retinopathy. These patients have a higher risk of having much more severe retinopathy too. The effects of hypothyroidism on platelet function and its rheological effect are said to play an important role in this regard. A higher prevalence of thyroid dysfunction (especially SCH) has been noted in the south and South EastAsians having Type 2 DM.

The effects of thyroid hormones on pathogenesis of diabetic retinopathy:

Numerous factors have been implicated in the pathogenesis of retinal new vessels including erythropoietin, growth hormone, Basic Fibroblast Growth Factor (bFGF), Insulin-like Growth Factor 1 (IGF-1) and VEGF. Other classes of molecules involved in pathogenesis of retinal new vessels other than growth factors include integrin are which act as specific extracellular matrix proteins. Integrin $\alpha v \beta 3$ is usually not expressed on microvessels, but is increased in response to angiogenic stimulation. New vessels in ocular tissue from patients with diabetic retinopathy especially proliferative diabetic retinopathy have shown selective up regulation of integrin $\alpha v \beta 3$ and $\alpha v \beta 5$.

Various studies have shown that pharmacologic inhibition of this integrin blocks new vessels in multiple animal models. Integrin $\alpha v \beta 3$ has proangiogenic effects on various growth factors including VEGF. On stimulation with VEGF, it interacts with Vascular Endothelial Growth Factor Receptor 2 (VEGFR2) and forms a complex with VEGFR2. Inhibition of integrin $\alpha v \beta 3$ results in decreased VEGFR2 autophosphorylation and signalling.

The molecular mechanism for proangiogenic activity of thyroid hormones is genomic and nongenomic. It is initiated nongenomically by integrin. Integrin $\alpha v \beta 3$ has cell surface receptor site for thyroid hormone for L-thyroxin (T4) that mediates proangiogenic action of thyroid hormones. ${ }^{7}$ Kinase transduction of the thyroid hormone signal and transcription of many angiogenic genes occurs.

\section{Limitations of this Study}

The relationship between thyroid dysfunction and severity of PDR could not be studied due to the small size of this study population.

Only hypertension and nephropathy were ruled out among the study population. Factors like dyslipidaemia, which are associated with both thyroid dysfunction and retinopathy were not ruled out.

Thyroid function tests did not include free T4. 


\section{CONCLUSION}

The prevalence of thyroid dysfunction among Type 2 Diabetes patients is $23 \%$. Subclinical hypothyroidism is the most common thyroid abnormality among Type 2 diabetics at $21 \%$. Prevalence of hypothyroidism in Type $2 \mathrm{DM}$ is $2 \%$ and that of hyperthyroidism is $0 \%$. Subclinical and overt hypothyroidism is associated with an increased risk of retinopathy.

One should have a high index of suspicion regarding the presence of thyroid dysfunction among Type 2 Diabetics. Routine screening for Thyroid dysfunction is recommended in all patients with retinopathy.

Further trials studying the impact of treatment of thyroid dysfunction on the complications of Diabetes need to be carried out.

\section{ACKNOWLEDGEMENTS}

We express our sincere thanks and gratitude to the Dean, HOD of Diabetology and HOD of Endocrinology of Government Rajaji Hospital and Madurai Medical College for permitting us to conduct this study for evaluation of their patients and their support to this study.

I am extremely grateful to all our Assistant Professors and PG Residents for their constant source of cheer and encouragement throughout the study. I thank all my patients who have formed the back bone of my study, without them this work would not have been possible. I am also thankful to all paramedical staff of all departments.

\section{REFERENCES}

1. Duntas LH, Orgiazzi J, Brabant G. The interface between thyroid and diabetes mellitus. Clin Endocrinol 2011;75(1):1-9.

2. Yang JK, Liu W, Shi J, et al. An association between subclinical hypothyroidism and sight-threatening diabetic retinopathy in type 2 diabetic patients. Diabetes Care. 2010;33(5):1018-20.

3. Davis PJ, Davis FB, Mousa SA. Thyroid hormone-induced angiogenesis. Curr Cardiol Rev. 2009;5(1):12-6.

4. Papazafiropoulou A, Sotiropoulos A, Kokolaki A, et al. Prevalence of thyroid dysfunction among Greek type 2 diabetic patients attending an outpatient clinic. J Clin Med Res. 2010;2(2):75-8.

5. Smithson MJ. Screening for thyroid dysfunction in a community population of diabetic patients. Diabet Med. 1998;15(2):148-50.

6. Masunaga R, Nagasaka A, Nakai A, et al. Alteration of platelet aggregation in patients with thyroid disorders. Metabolism. 1997;46(10):1128-31.

7. Davis FB, Mousa SA, O'Connor L, et al. Proangiogenic action of thyroid hormone is fibroblast growth factordependent and is initiated at the cell surface. Circ Res. 2004;94(11):1500-6. 